\title{
Performance of multiparametric MRI appears better when measured in patients who undergo radical prostatectomy
}

This article was published in the following Dove Press journal:

Research and Reports in Urology

\author{
Nancy N Wang' \\ Richard E Fan' \\ John T Leppert ${ }^{1,2}$ \\ Pejman Ghanouni ${ }^{3}$ \\ Christian A Kunder ${ }^{4}$ \\ James D Brooks' \\ Benjamin I Chung' \\ Geoffrey A Sonn ${ }^{1,3}$ \\ 'Department of Urology, Stanford \\ University School of Medicine, \\ Stanford, CA, USA; ${ }^{2}$ Veterans Affairs, \\ Palo Alto Health Care System, Palo \\ Alto, CA, USA; ${ }^{3}$ Department of \\ Radiology, Stanford University School \\ of Medicine, Stanford, CA, USA; \\ ${ }^{4}$ Department of Pathology, Stanford \\ University School of Medicine, \\ Stanford, CA, USA
}

Correspondence: Nancy N Wang Department of Urology, Stanford University School of Medicine, 300 Pasteur Drive, S287, Stanford, CA 94304 , USA

Tel +I 6507935585

$\mathrm{Fax}+\mathrm{I} 6504985346$

Email Nwang4@stanford.edu

\begin{abstract}
Utilization of pre-biopsy multiparametric MRI (mpMRI) is increasing. To optimize the usefulness of mpMRI, physicians should accurately quote patients a numerical risk of cancer based on their MRI. The Prostate Imaging Reporting and Data System (PIRADS) standardizes interpretation of mpMRI; however, reported rates of clinically significant prostate cancer (CSC) stratified by PIRADS score vary widely. While some publications use radical prostatectomy (RP) specimens as gold standard, others use biopsy. We hypothesized that much of the variation in CSC stems from differences in cancer prevalence in RP cohorts $(100 \%$ prevalence) vs biopsy cohorts. To quantify the impact of this selection bias on cancer yield according to PIRADS score, we analyzed data from 614 men with 854 lesions who underwent targeted biopsy from 2014 to 2018 . Of these, 125 men underwent RP. We compared the PIRADS detection rates of CSC (Gleason $\geq 7$ ) on targeted biopsy between the biopsy-only and RP cohorts. For all PIRADS scores, CSC yield was much greater in patients who underwent RP. For example, CSC was found in $30 \%$ of PIRADS 3 lesions in men who underwent RP vs $7.6 \%$ in men who underwent biopsy. Our results show that mpMRI performance appears to be better in men who undergo RP compared with those who only receive biopsy. Physicians should understand the effect of this selection bias and its magnitude when discussing mpMRI results with patients considering biopsy, and take great caution in quoting CSC yields from publications using RP as gold standard.
\end{abstract}

Keywords: counseling, image-guided biopsy, magnetic resonance imaging, patient selection, prostatic neoplasms

\section{Introduction}

Multiparametric MRI (mpMRI) has been widely adopted in the diagnosis and management of prostate cancer based on landmark studies demonstrating high sensitivity for detection of clinically significant prostate cancer (CSC) ${ }^{1-3}$ Ideally, mpMRI could simplify decisions about biopsy. However, CSC yield on mpMRI varies widely in the literature. When stratified by Prostate Imaging Reporting and Data System (PIRADS) scores, the detection of CSC ranges from $0 \%$ to $66 \%$ for PIRADS 3 lesions, ${ }^{2,4,5} 21 \%$ 98\% for PIRADS 4, 2,4,6 and 75\%-99\% for PIRADS 5.,4,6 This variation complicates patient counseling. While differences in image acquisition and radiologist interpretation can affect the predictive value of mpMRI, ${ }^{7}$ another factor that is often mentioned but not previously quantified is the impact of cancer prevalence in the study population.

Most studies evaluating mpMRI accuracy utilize one of two cohorts: 1) men who undergo MRI and targeted biopsy with biopsy histopathology as the reference, or 2) men who undergo MRI and radical prostatectomy (RP) with surgical pathology as 
the reference. While biopsy cohorts are larger and include patients with low-grade or benign pathology, sampling error may lead to under-diagnosis of CSC, even with targeted techniques. ${ }^{8}$ In contrast, while prostatectomy cohorts have gold standard pathology, this population has $100 \%$ cancer prevalence and therefore is not representative of men considering biopsy. ${ }^{9}$ Therefore, reported variation in CSC yield across PIRADS scores may be largely due to population differences between prostatectomy and biopsy-only cohorts.

Although several studies mention the presence of this selection bias, ${ }^{1,6,9,10}$ the magnitude of its effects on CSC detection rates has not been previously quantified. We used a large prospectively collected MRI-targeted biopsy database to measure the difference in CSC yield, stratified by PIRADS score, between biopsy-only and prostatectomy cohorts.

\section{Materials and methods}

We evaluated 614 consecutive men who underwent $3 \mathrm{~T}$ mpMRI followed by targeted biopsy at our institution between March 2014 and January 2018. Of these, 409 were included in a prior publication. ${ }^{7}$ Following Stanford IRB approval, written informed consent for data collection was obtained prior to biopsy. The study and protocol were in accordance with Declaration of Helsinki principles. We excluded patients who were previously treated for prostate cancer or had a normal mpMRI. We evaluated the first biopsy for men who underwent more than one biopsy. Reporting was in accordance with START guidelines where applicable. ${ }^{11}$

Attending radiologists with expertise in body imaging interpreted all mpMRI images. Targeted biopsies were performed by urologists using the Artemis robotic biopsy device (Eigen, Grass Valley, CA, USA) following a standard protocol. Targeted biopsy histopathology was the reference standard used for both cohorts to allow for direct comparison of CSC yield. Prostatectomy pathology was not used because it was not available for the biopsy-only patients. To exclude overlap error, we compared the results of men who underwent targeted biopsy only with the prostatectomy group through a multivariable logistic regression analysis clustered by patient using STATA 14 (StataCorp LP, College Station, TX, USA).

\section{Results}

The biopsy-only cohort consisted of 489 men with 689 lesions. Median age was 65 years with median PSA of $7.5 \mathrm{ng} / \mathrm{mL}$. The prostatectomy cohort consisted of 125 men with 165 lesions. Median age was 65 years with median PSA of $9 \mathrm{ng} / \mathrm{mL}$ (Table 1). CSC was detected in $75 \%$ of lesions in the prostatectomy group and $27 \%$ of lesions in the biopsy-only cohort (Table 1).

The CSC detection rate in the RP cohort was 90\% for PIRADS 5 lesions, 58\% for PIRADS 4, 30\% for PIRADS $3 \%$, and $45 \%$ for PIRADS 2 compared to $56 \%, 31 \%, 7.6 \%$, and $10 \%$ in the biopsy-only cohort (Table 1). The difference in detection is present for all PIRADS scores. After adjusting for PIRADS, PSA, age, and race, and clustered by patient to control for multiple lesions within a patient, the detection of CSC was still significantly higher in the prostatectomy cohort compared with the biopsy-only cohort with an overall OR of 4.26 (95\% CI 2.67-6.81).

\section{Discussion}

We found that the CSC yield was much greater for all PIRADS scores in men who subsequently underwent RP.

Table I Demographic, clinical, and biopsy results for men who underwent multiparametric MRI and targeted biopsy, separated into a biopsy-only cohort and radical prostatectomy cohort

\begin{tabular}{|c|c|c|c|}
\hline & Full study group $(\mathrm{N}=6 \mid 4)$ & Biopsy only $(\mathrm{N}=489)$ & $\begin{array}{l}\text { Radical } \\
\text { prostatectomy }(\mathrm{N}=\mid 25)\end{array}$ \\
\hline Median age (years) & 65 (IQR 60-69) & 65 (IQR 60-70) & 65 (IQR 60-68) \\
\hline Median PSA & $8 \mathrm{ng} / \mathrm{mL}$ (IQR 5.5-II.5) & $7.5 \mathrm{ng} / \mathrm{mL}$ (IQR 5.3-II.3) & $9 \mathrm{ng} / \mathrm{mL}(\mathrm{IQR} 6 . \mathrm{I}-\mathrm{I} 2.9)$ \\
\hline \multicolumn{4}{|l|}{ Race (\%) } \\
\hline White & $421(69 \%)$ & $328(67 \%)$ & $93(74 \%)$ \\
\hline Asian/Native Indian & $76(12 \%)$ & $59(12 \%)$ & $17(14 \%)$ \\
\hline Unknown & $56(9 \%)$ & $50(10 \%)$ & $6(5 \%)$ \\
\hline Hispanic & $36(6 \%)$ & $31(6 \%)$ & $5(4 \%)$ \\
\hline Black & $25(4 \%)$ & $21(4 \%)$ & $4(3 \%)$ \\
\hline Total lesions & 854 & 689 & 165 \\
\hline CSC lesions (\%) & $288(34 \%)$ & $189(27 \%)$ & $99(75 \%)$ \\
\hline PIRADS 5 & $122(65 \%)$ & $76(56 \%)$ & $46(90 \%)$ \\
\hline PIRADS 4 & $127(36 \%)$ & $90(31 \%)$ & $37(58 \%)$ \\
\hline PIRADS 3 & $26(11 \%)$ & $15(7.6 \%)$ & II (30\%) \\
\hline PIRADS 2 & II (I5\%) & $6(10 \%)$ & $5(45 \%)$ \\
\hline
\end{tabular}

Abbreviations: CSC, clinically significant cancer (Gleason $\geq 3+4$ ); PIRADS, Prostate Imaging Reporting and Data System. 
Though this finding is intuitive, our study is the first to quantify the effect that patient cohort (biopsy vs prostatectomy) has on mpMRI detection of CSC. In order to provide relevant statistics to patients considering biopsy, it is crucial for clinicians to understand the methods used by studies evaluating prostate mpMRI. Those studies using prostatectomy gold standard will overestimate the CSC yield for a man who is considering biopsy and may be clinically misleading. For example, a patient with a PIRADS 3 lesion may decide differently if he is quoted a $7.6 \%$ vs $30 \%$ probability of CSC on targeted biopsy (Table 1).

Our study has several notable strengths. First, all mpMRIs were evaluated prior to biopsy, with no knowledge of each patient's final treatment plan. Second, all targeted biopsies were performed by one of two urologists with extensive experience. Third, all patients were recruited through routine clinical workflow and were comparable in age and racial makeup. Fourth, we compared biopsy histopathology instead of incorporating whole-mount pathology for the prostatectomy group, which could have eliminated sampling error in only one cohort.

Weaknesses include limited generalizability, as this is a single-site study, and lack of complete information on patients treated outside of our facility. We also retrospectively separated men with prostatectomy from those without to conduct the statistical analysis, which may artificially increase the differences in cancer yield. To mitigate this, we presented descriptive results for our full study group (Table 1). We also recognize that variation exists among radiologists interpreting mpMRI; ${ }^{7}$ however, it is unlikely that this would account for the large difference seen, as radiologists were randomly distributed across both cohorts. Finally, it should be noted that transperineal template mapping biopsy studies may have less patient selection bias, as they utilize a biopsy population while more accurately capturing whole-gland pathology compared to targeted biopsy. ${ }^{1,10}$

\section{Conclusion}

This study is the first to explicitly quantify the effect of patient selection on prostate mpMRI performance. The predictive strength of PIRADS scores vary depending on the cancer prevalence in the population and cannot be viewed in isolation. Our findings provide an important perspective for clinicians to consider when evaluating mpMRI diagnostic accuracy and counseling patients. Additionally, when considering mpMRI machine-learning initiatives, our results show that patient selection and choice of pathologic reference standard are important considerations.

\section{Disclosure}

The authors report no conflicts of interest in this work.

\section{References}

1. Ahmed HU, El-Shater Bosaily A, Brown LC, et al. Diagnostic accuracy of multi-parametric MRI and TRUS biopsy in prostate cancer (PROMIS): a paired validating confirmatory study. Lancet. 2017;389(10071): 815-822.

2. Kasivisvanathan V, Rannikko AS, Borghi M, et al. MRI-targeted or standard biopsy for prostate-cancer diagnosis. N Engl J Med. 2018;378(19): 1767-1777.

3. Meng X, Rosenkrantz AB, Mendhiratta N, et al. Relationship between prebiopsy multiparametric magnetic resonance imaging (MRI), biopsy indication, and mri-ultrasound fusion-targeted prostate biopsy outcomes. Eur Urol. 2016;69(3):512-517.

4. Mertan FV, Greer MD, Shih JH, et al. Prospective evaluation of the prostate imaging reporting and data system version 2 for prostate cancer detection. J Urol. 2016;196(3):690-696.

5. Cash H, Maxeiner A, Stephan C, et al. The detection of significant prostate cancer is correlated with the prostate imaging reporting and data system (PI-RADS) in MRI/transrectal ultrasound fusion biopsy. World J Urol. 2016;34(4):525-532.

6. Lim CS, Mcinnes MDF, Lim RS, et al. Prognostic value of prostate imaging and data reporting system (PI-RADS) v. 2 assessment categories 4 and 5 compared to histopathological outcomes after radical prostatectomy. J Magn Reson Imaging. 2017;46(1):257-266.

7. Sonn GA, Fan RE, Ghanouni P, et al. Prostate magnetic resonance imaging interpretation varies substantially across radiologists. Eur Urol Focus. Epub 2017 Dec 06.

8. Siddiqui MM, Rais-Bahrami S, Turkbey B, et al. Comparison of MR/ ultrasound fusion-guided biopsy with ultrasound-guided biopsy for the diagnosis of prostate cancer. JAMA. 2015;313(4):390-397.

9. El-Shater Bosaily A, Parker C, Brown LC, et al. PROMIS-Prostate MR imaging study: a paired validating cohort study evaluating the role of multi-parametric MRI in men with clinical suspicion of prostate cancer. Contemp Clin Trials. 2015;42:26-40.

10. Wysock JS, Lepor H. Multi-parametric MRI imaging of the prostateimplications for focal therapy. Transl Androl Urol. 2017;6(3):453-463.

11. Moore CM, Kasivisvanathan V, Eggener S, et al. Standards of reporting for MRI-targeted biopsy studies (START) of the prostate: recommendations from an International Working Group. Eur Urol. 2013;64(4):544-552.
Research and Reports in Urology

Publish your work in this journal

Research and Reports in Urology is an international, peer-reviewed, open access journal publishing original research, reports, editorials, reviews and commentaries on all aspects of adult and pediatric urology in the clinic and laboratory including the following topics: Pathology, pathophysiology of urological disease; Investigation and treatment of

\section{Dovepress}

urological disease; Pharmacology of drugs used for the treatment of urological disease. The manuscript management system is completely online and includes a very quick and fair peer-review system, which is all easy to use. Visit http://www.dovepress.com/testimonials.php to read real quotes from published authors. 